\title{
New mutational trends in the HA protein of 2009 H1N1 pandemic influenza virus from May 2010 to February 2011
}

Wei Hu

Department of Computer Science, Houghton College, Houghton, USA; wei.hu@houghton.edu

Received 2 March 2011; revised 5 April 2011; accepted 21 April 2011.

\section{ABSTRACT}

As we enter the year of 2011, the 2009 H1N1 pandemic influenza virus is in the news again. At least 20 people have died of this virus in China since the beginning of 2011 and it is now the predominant flu strain in the country. Although this novel virus was quite stable during its run in the flu season of 2009-2010, a genetic variant of this virus was found in Singapore in early 2010, and then in Australia and New Zealand during their 2010 winter influenza season. Several critical mutations in the HA protein of this variant were uncovered in the strains collected from January 2010 to April 2010. Moreover, a structural homology model of HA from the $A / B r i s b a n e / 10 / 2010(H 1 N 1)$ strain was made based on the structure of $A / C a$ alifornia/04/2009 (H1N1). The purpose of this study was to investigate mutations in the HA protein of 2009 H1N1 from sequence data collected worldwide from May 2010 to February 2011. A fundamental problem in bioinformatics and biology is to find the similar gene sequences for a given gene sequence of interest. Here we proposed the inverse problem, i.e., finding the exemplars from a group of related gene sequences. With a clustering algorithm affinity propagation, six exemplars of the HA sequences were identified to represent six clusters. One of the clusters contained strain $A / B$ risbane/12/2010(H1N1) that only differed from $A / B r i s b a n e / 10 / 2010$ in the HA sequence at position 449. Based on the sequence identity of the six exemplars, nine mutations in HA were located that could be used to distinguish these six clusters. Finally, we discovered the change of correlation patterns for the HA and NA of 2009 H1N1 as a result of the HA receptor binding specificity switch, revealing the balanced interplay between these two surface proteins of the virus.

Keywords: 2009 H1N1; Affinity Propagation; Clustering Algorithms; Entropy; Exemplars; Hemagglutinin; Influenza; Informational Spectrum Method; Mutation; Mutual Information; Receptor Binding Specificity

\section{INTRODUCTION}

A report on February 10, 2011 indicated that 2009 H1N1 has become the predominant strain of influenza virus in China and caused at least twenty deaths in the country since the beginning of 2011 [1]. As the 20102011 flu season continues, this virus remains a public health concern. This novel swine-origin virus maintained its genetic stability during its run in the 2009-2010 flu season for the most part. However, a genetic variant of this virus was found first in Singapore, and then in Oceania including Australia and New Zealand during the southern hemisphere 2010 winter. Several vaccine breakthroughs and fatal cases might be linked to this variant [2]. These new developments call for a continued surveillance of its circulation and evolution, even though extensive research on 2009 H1N1 has been conducted including a series of studies in [3-13].

Several critical mutations including D94N, N125D, and $\mathrm{V} 250 \mathrm{~A}$ in the HA protein of this variant were discovered in the strains collected from January 2010 to April 2010. Furthermore, structural homology model of HA from the A/Brisbane/10/2010 strain was created based on the $\mathrm{A} / \mathrm{California} / 04 / 2009$ structure. Interestingly, two mutations D94N and V250A tended to occur in pairs [2,14]. The bioinformatics analysis in [13] suggested that the reason for this pairing was to allow the virus to maintain HA binding to human type receptor, so the virus could replicate in humans efficiently. Using this interpretation as guide, a new mutation V30A was 
uncovered in the strains from Singapore that could compensate for N125D as V250A did for D94N. These mutations were found to cause the HA receptor binding specificity shift from A/California/07/2009, a strain used in the vaccine for $2009 \mathrm{H} 1 \mathrm{~N} 1$.

After the discovery of this new variant, the natural question is what happened next to this virus. Our interest in this study was to expand the work started in [13] to examine the HA mutations in the strains of $2009 \mathrm{H} 1 \mathrm{~N} 1$ collected after 05-01-2010 and compared them to those collected before 05-01-2010.

Due to the enormous global efforts, large number of 2009 H1N1 sequences have been collected. One challenge facing us today is how to extract useful information and knowledge for this virus from this huge database of sequences. A fundamental problem in bioinformatics and biology is to find the similar gene sequences for a given gene sequence of interest. Here we asked the inverse problem: given a collection of related gene sequences, what are the exemplars of these sequences. In the present study, a clustering strategy affinity propagation [15] was employed to find exemplars of the HA sequences of $2009 \mathrm{H} 1 \mathrm{~N} 1$ collected between 05-01-2010 and 02-22-2011. These exemplars served as the starting point to uncover the mutations in the HA protein of 2009 H1N1 after 05-01-2010.

The HA protein of $2009 \mathrm{H} 1 \mathrm{~N} 1$ exhibits dual receptor binding preference $[11,16]$, which prompted us to explore the effects of binding preference change on NA because the balance between host cell binding and sialic acid receptor destruction needs to be maintained by HA and NA. With the large number of 2009 H1N1 sequences collected to date, it is now possible to conduct bioinformatics analysis to probe this kind of interactions between HA and NA. Therefore, another task of our study is to investigate the cooperation between the HA and NA of $2009 \mathrm{H} 1 \mathrm{~N} 1$ in the process of HA receptor binding affinity shift.

\section{MATERIALS AND METHODS}

\subsection{Sequence Data}

Protein sequences of influenza were retrieved from the EpiFlu Database (http://platform.gisaid.org) of GISAID. Only the full length and unique sequences were selected. All sequences used in this study were aligned with MAFFT [17].

\subsection{Affinity Propagation}

At present, several clustering algorithms such as Kmeans use data to find centers of clusters. The novelty of affinity propagation clustering algorithm $[15,18]$ lies in the search of exemplars from data to represent clusters through message passing. The data points in a cluster connects to the exemplar that best represents it.

Affinity propagation (AP) was designed to searches for a set of exemplars from $n$ data points so the minima of the following energy function could be achieved

$$
E(e)=-\sum_{i=1}^{n} s\left(i, e_{i}\right) \quad s\left(i, e_{i}\right) \leq 0
$$

where $e_{i}$ represents the exemplar of data point $i$, and $s\left(i, e_{i}\right)$ is the similarity between data point $i$ and its exemplar $e_{i}$. In general, finding an optimal set of exemplars from data is a computationally intensive endeavor. However, the identification of these exemplars in AP were accomplished with an efficient algorithm that employs real-valued messages exchanged among data points. There were two types of messages, responsibility and availability. The responsibility $r(i, k)$, sent from data point $i$ to candidate exemplar point $k$, reflects the suitability for point $k$ to be the exemplar for point $i$. The availability $a(i, k)$, sent from candidate exemplar point $k$ to point $i$, represents the propensity for point $i$ to choose point $k$ as its exemplar. In this study, the similarity used as input to AP is the Hamming distance between two sequences. At the beginning of affinity propagation the availability matrix is set to zero, and then repeat the following iteration until a stopping criterion is satisfied:

$$
\begin{gathered}
r(i, k) \leftarrow s(i, k)-\max _{k^{\prime} \text { s.t. } k^{\prime} \neq k}\left\{a\left(i, k^{\prime}\right)+s\left(i, k^{\prime}\right)\right\} \\
a(i, k) \leftarrow \min \left\{0, r(k, k)+\sum_{i^{\prime} \text { s.t. } i^{\prime} \neq i, k} \max \left\{0, r\left(i^{\prime}, k\right)\right\}\right\} \\
a(k, k) \leftarrow \sum_{i^{\prime} \text { s.t. } i^{\prime} \neq k} \max \left\{0, r\left(i^{\prime}, k\right)\right\}
\end{gathered}
$$

At each iteration, the messages indicate the current affinity between data points and their exemplars. After this original AP model, a binary variable model for affinity propagation was proposed in [19], and several applications of AP could be found in [20-22].

\subsection{Informational Spectrum Method}

The informational spectrum method (ISM) is a bioinformatics technique that can be used to analyze protein sequences [23-27]. The idea is to translate the protein sequences into numerical sequences based on electron-ion interaction potential (EIIP) of each amino acid. Then the Discrete Fourier Transform (DFT) can be applied to these numerical sequences, and the resulting DFT coefficients are used to produce the energy density spectrum. The informational spectrum (IS) comprises the frequencies and the amplitudes of this energy density spectrum. According to the ISM theory, the peak frequencies of IS of a protein sequence reflect its biological or biochemical functions. The ISM was successfully 
applied to quantify the effects of HA mutations on the receptor binding preference in [8] and reveal the change of receptor binding selection caused by the mutations identified in [13]. Table 1 shows several common IS frequencies identified in $[23,24]$.

It was observed in $[11,16]$ that some of the strains of 2009 H1N1 displayed dual HA receptor binding preference. Consequently, in this study we used top two IS frequencies, one primary and one secondary, to describe the HA receptor selection.

\subsection{Entropy and Mutual Information}

In information theory [28,29], entropy is a measure of the uncertainty associated with a random variable. Let $x$ be a discrete random variable that has a set of possible values $\left\{a_{1}, a_{2}, a_{3}, \cdots, a_{n}\right\}$ with probabilities $\left\{p_{1}, p_{2}, p_{3}, \cdots, p_{n}\right\}$ where $P\left(x=a_{i}\right)=p_{i}$. The entropy $H$ of $x$ is

$$
H(x)=-\sum_{i} p_{i} \log p_{i}
$$

The mutual information of two random variables is a quantity that measures the mutual dependence of the two variables or the average amount of information that $x$ conveys about $y$, which can defined as

$$
I(x, y)=H(x)+H(y)-H(x, y)
$$

where $H(x)$ is the entropy of $x$, and $H(x, y)$ is the joint entropy of $x$ and $y . I(x, y)=0$ if and only if $x$ and $y$ are independent random variables.

In the current study, each of the $n$ columns in a multiple sequence alignment of a set of influenza protein sequences of length $N$ is considered as a discrete random variable $x_{i}(1 \leq i \leq N)$ that takes on one of the $20(n=20)$ amino acid types with some probability. $H\left(x_{i}\right)$ has its minimum value 0 if all the amino acids at position $i$ are the same, and achieves its maximum if all the 20 amino acid types appear with equal probability at position $i$, which can be verified by the Lagrange multiplier technique. A position of high entropy means that the amino acids are often varied at this position. While $H\left(x_{i}\right)$ measures the genetic diversity at position $i$ in our current study, $I\left(x_{i}, y_{i}\right)$ measures the correlation between amino acid substitutions at positions $i$ and $j$.

\section{RESULTS}

\subsection{Mutations in HA and NA of $2009 \mathrm{H} 1 \mathrm{~N} 1$ after 05-01-2011}

With a clustering technique affinity propagation [15], we were able to find six exemplars of the HA sequences of $2009 \mathrm{H} 1 \mathrm{~N} 1$ collected between 05-01-2010 and 02-222011, representing six different clusters (Table 2). Mutations at nine positions 97, 125, 134, 183, 185, 295, 374, 441, and 451 in the HA protein were identified, which could be used to distinguish these exemplars and clusters. One of them happened to be N125D (Figure 1), a mutation found in the genetic variant of 2009 H1N1 in Singapore, Australia and New Zealand [2]. The HA active site is located in a cleft, which is lined by several conserved residues $91,150,152,180,187,191$, and 192, and the cleft is formed by its right edge (131_GVTAA) and left edge (221_RGQAGR) [30]. It was worth noting that three of the nine mutation sites, 134, 183, and 185 , were close to some of these critical sites in HA. Cluster 1 contained a strain A/Brisbane/12/2010, which is different from the model strain A/Brisbane/10/2010 in [2]

Table 1. Characteristic IS frequencies of HA proteins in $2009 \mathrm{H} 1 \mathrm{~N} 1$, swine H1N1/H1N2, avian H1N1, and A/South Carolina/1/18 (H1N1).

\begin{tabular}{ccccc}
\hline Subtype & 2009 H1N1 & Swine H1N2/H1N1 & Avian H1N1 & A/South Carolina/1/18 (H1N1) \\
\hline Frequency & $\mathrm{F}(0.295)$ & $\mathrm{F}(0.055)$ & $\mathrm{F}(0.282)$ & $\mathrm{F}(0.258)$ \\
\hline
\end{tabular}

Table 2. Meta information for the six exemplars and six clusters from the HA sequences of 2009 H1N1 between 05-01-2010

\begin{tabular}{|c|c|c|c|c|}
\hline Cluster Number & Primary Frequency & Secondary Frequency & Cluster Size & Exemplar Strain ID \\
\hline Cluster 1 & 0.258 & 0.295 & 21 & A/Navarra/RR6914/10 \\
\hline Cluster 2 & 0.295 & 0.055 & 44 & A/Bangladesh/5557/2010 \\
\hline Cluster 3 & 0.295 & 0.258 & 12 & A/Guatemala/287/2010 \\
\hline Cluster 4 & 0.295 & 0.055 & 15 & A/Madrid/SO8034/10 \\
\hline Cluster 5 & 0.295 & 0.282 & 17 & A/Pennsylvania/34/2010 \\
\hline Cluster 6 & 0.295 & 0.258 & 30 & A/Vaxjo/2/2010 \\
\hline
\end{tabular}
and $02-22-2011$. 

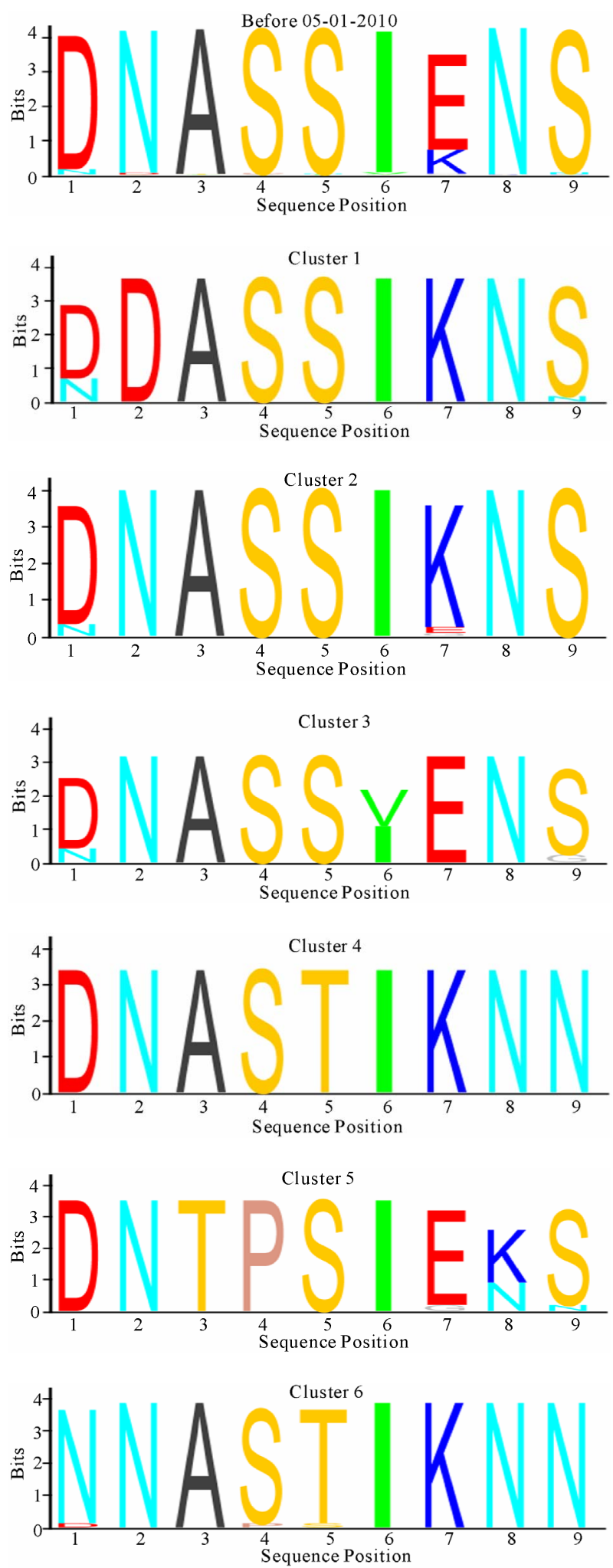

Figure 1. Amino acid logo at positions 97, 125, 134, 183, 185, $295,374,441$ and 451 in the HA sequences of the six clusters after 05-01-2010 and the HA sequences before 05-01-2010. These nine positions are ordered as $1,2,3,4,5,6,7,8$ and 9 in the figure. by only one amino acid at position 449, and this cluster carried the signature mutation N125D.

The different HA binding preferences of the six clusters as primary IS frequency and secondary frequency were calculated, manifesting the impact of the nine mutations on receptor selection (Table 2). The analysis in [13] studied the HA receptor specificity of 2009 H1N1 as a function of time, which showed that the virus demonstrated swine receptor preference in the early months of its course, and this preference gradually disappeared in the late months as a whole group. But there were strains, those in clusters 2 and 4, collected after 05-012010 that still displayed swine receptor selection at frequency $\mathrm{F}(0.055)$ (Table 2).

It is well-known that HA and NA depend on each other. To reveal the influence of the nine mutations in HA on the NA protein, we also looked into the corresponding NA sequences. There were four positions 241 , 366,369 , and 394 in the consensus NA sequences of the six clusters that had different amino acids, implying less variation in NA compared to HA (Figure 2). The Hamming distances of the six consensus HA amino acid sequences after 05-01-2010 from that before 05-01-2010 were in the range of 1 to 4, whereas the same Hamming distances for NA were in the range of 0 to 2 (Figures 1 and 2). The amino acid logos in Figures 1 and 2 illustrated the consensus and variation of the amino acids at these selected positions in HA and NA.

The active site of NA is lined by several conserved residues $(117-119,133-138,146-152,156,179,180$, 196-200, 223-228, 243-247, 277, 278, 293, 295, 344-347, $368,401,402$, and 426-441) that participate in recognition of its substrate [31]. Three of the four mutations in NA, 241, 366, and 369, were near some of these key residues. In [32] the role of second active site in NA was assessed. It found that in avian NA the interaction between this second site and the primary site is essential for NA function, and the NA of $2009 \mathrm{H} 1 \mathrm{~N} 1$ has retained some of the important features of the second site. Following [32], we listed the amino acids at the positions that are lined with the second site (N2 numbering) (Table 3). It appeared that the major difference in amino acids occurred at position 372 for the six clusters and A/California/04/2009(H1N1) (Cal_04_09).

The entropy at a position in an alignment of protein sequences measures the propensity of amino acids to change at that position. To gain a global perspective of $\mathrm{HA}$ and NA sequence variation before and after 05-012010, the entropy of these proteins was calculated (Figure 3). Furthermore, top 25 entropy positions in each protein were selected (Figure 4). As a general trend, the number of positive entropy positions before 05-01-2010 was larger than after 05-01-201. However, the magni- 

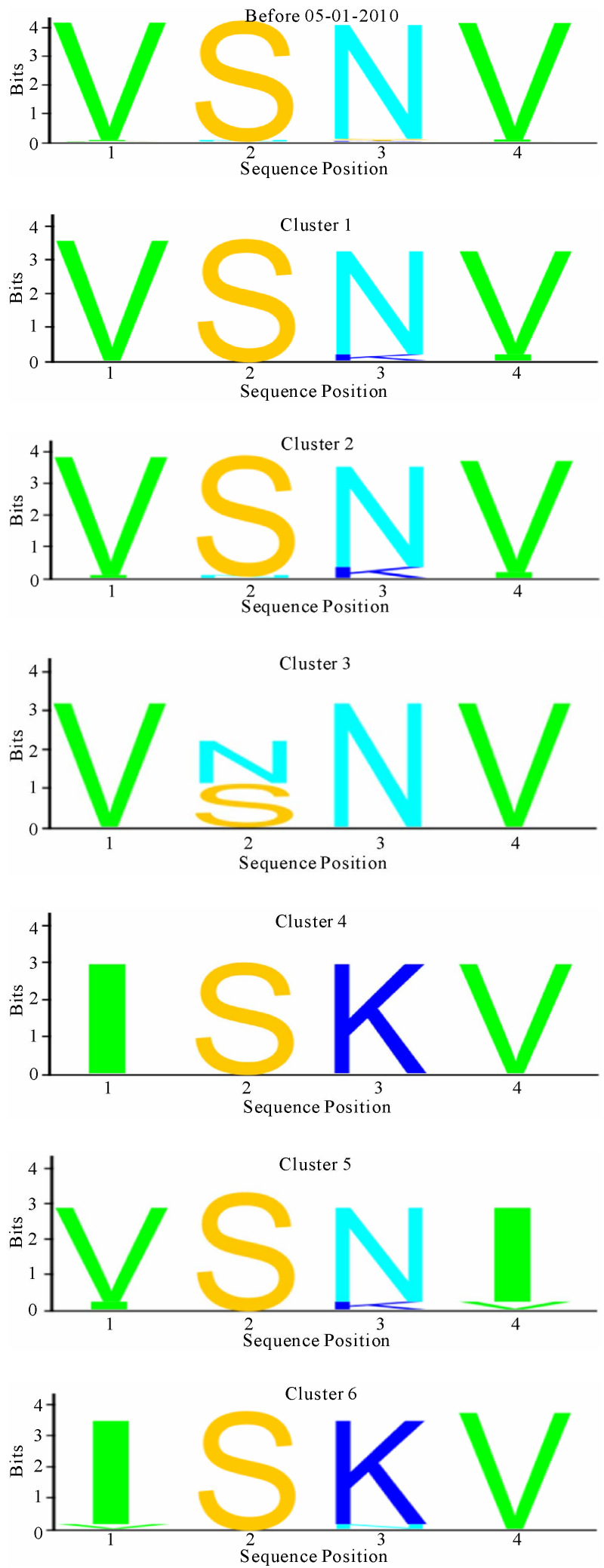

Figure 2. Amino acid logo at positions 241, 366, 369, and 394 in the NA sequences of the six clusters after 05-01-2010 and the NA sequences before 05-01-2010. These four positions are ordered as $1,2,3$, and 4 in the figure.
Table 3. Amino acid comparison at second active site residues in avian $\mathrm{N} 1$, human $\mathrm{N} 2$, and $\mathrm{N} 1$ of the six clusters and Cal_04_09. The conserved residues are highlighted (N2 numbering).

\begin{tabular}{cccc}
\hline & $366-373$ & $399-403$ & $430-433$ \\
\hline Avian N1 & KSTNSRSG & AITDWS & RPKE \\
Human N2 & ISKDLRSG & DSDNRS & RKQE \\
Cal_04_09 & KSISSRNG & GINEWS & RPKE \\
Cluster 1 & KSISSRNG & GIKEWS & RPKE \\
Cluster 2 & KSISSRNG & GINEWS & RPKE \\
Cluster 3 & KSINSRNG & GVNEWS & RPKE \\
Cluster 4 & KSISSRKG & GINEWS & RPKE \\
Cluster 5 & KSISSRNG & GINEWS & RPKE \\
Cluster 6 & KSISSRKG & GINEWS & RPKE \\
\hline
\end{tabular}

tude of the few top entropy positions after 05-01-2010 was bigger than before 05-01-2010. Within HA the entropy in the HA1 domain was higher than HA2 domain, and within NA the entropy near the stalk region was very high and the entropy in that region was much higher before than after 05-01-2010. The NA stalk length was demonstrated to be important for enhanced viral pathogenicity $[4,33,34]$.

Also a few top entropy positions in HA and NA were shared before and after 05-01-2010 (Figure 4). There were eight such positions in HA and 13 positions in NA. It was evident that all the nine mutation positions in HA and four in NA were among the top entropy positions in Figure 4. It was of note that two of the nine positions in HA, 374 and 451, had high entropy both before and after 05-01-2010. As the time moved from before 05-01-2010 to after 05-01-2010, the HA entropy increased at position 451 while decreased at position 222, and position 374 remained the highest entropy. During the same period, the NA entropy increased at positions 241 and 369 and became the top two entropy position after 05-012010. Three of the four NA mutation positions, 241, 366, and 396, had high entropy both before and after 05-012010.

\subsection{Correlation Patterns for HA and NA of 2009 H1N1 before and after HA Receptor Binding Affinity Shift}

The large number of influenza sequences collected during the pandemic 2009 provided an ideal opportunity for learning the interdependence between HA and NA in the shift of receptor binding preference. As seen in Table 2, the main HA binding frequencies of $2009 \mathrm{H} 1 \mathrm{~N} 1$ were $F(0.295), F(0.055), F(0.2823)$, and $F(0.2588)$. To inspect 

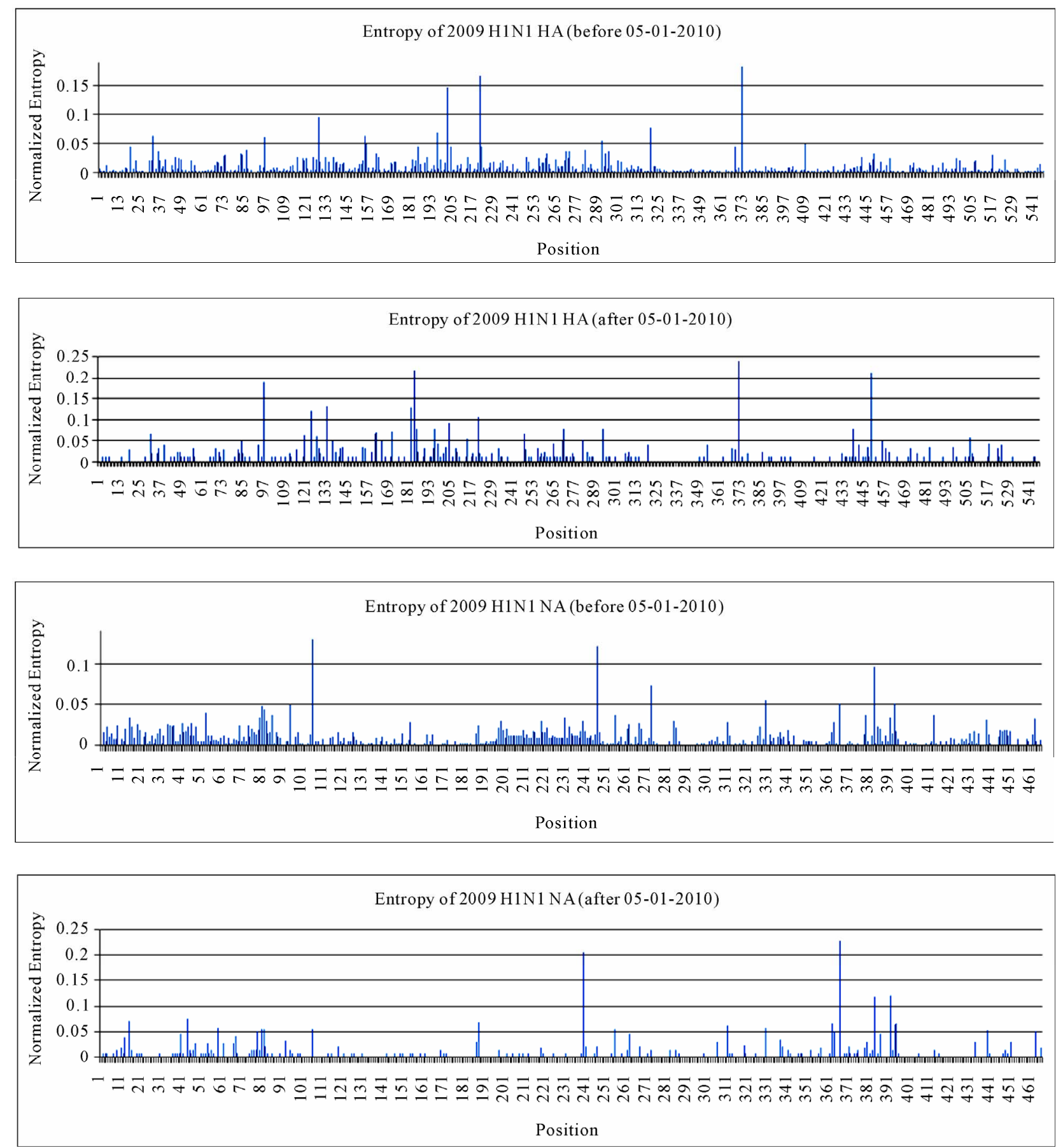

Figure 3. Entropy distribution of 2009 H1N1 HA and NA before and after 05-01-2010.

the variation of correlation patterns within and between HA and NA caused by receptor switch, we divided the whole set of HA sequences of $2009 \mathrm{H} 1 \mathrm{~N} 1$ into six groups according to their primary and secondary IS frequencies as shown in Table 4 and Figure 5. The HA sequences that had different frequency combinations from those listed in Figure 5 were not included in this analysis. As a measure of interactions among residues, we counted the number of two residues that had a positive mutual information value within and between HA and NA. Because we were interested in the relative counts within a group, the correlated pair counts were averaged by the number of sequences in each group to display them within one figure.

There was an apparent change of correlation patterns before and after the switch of receptor binding specificity. 

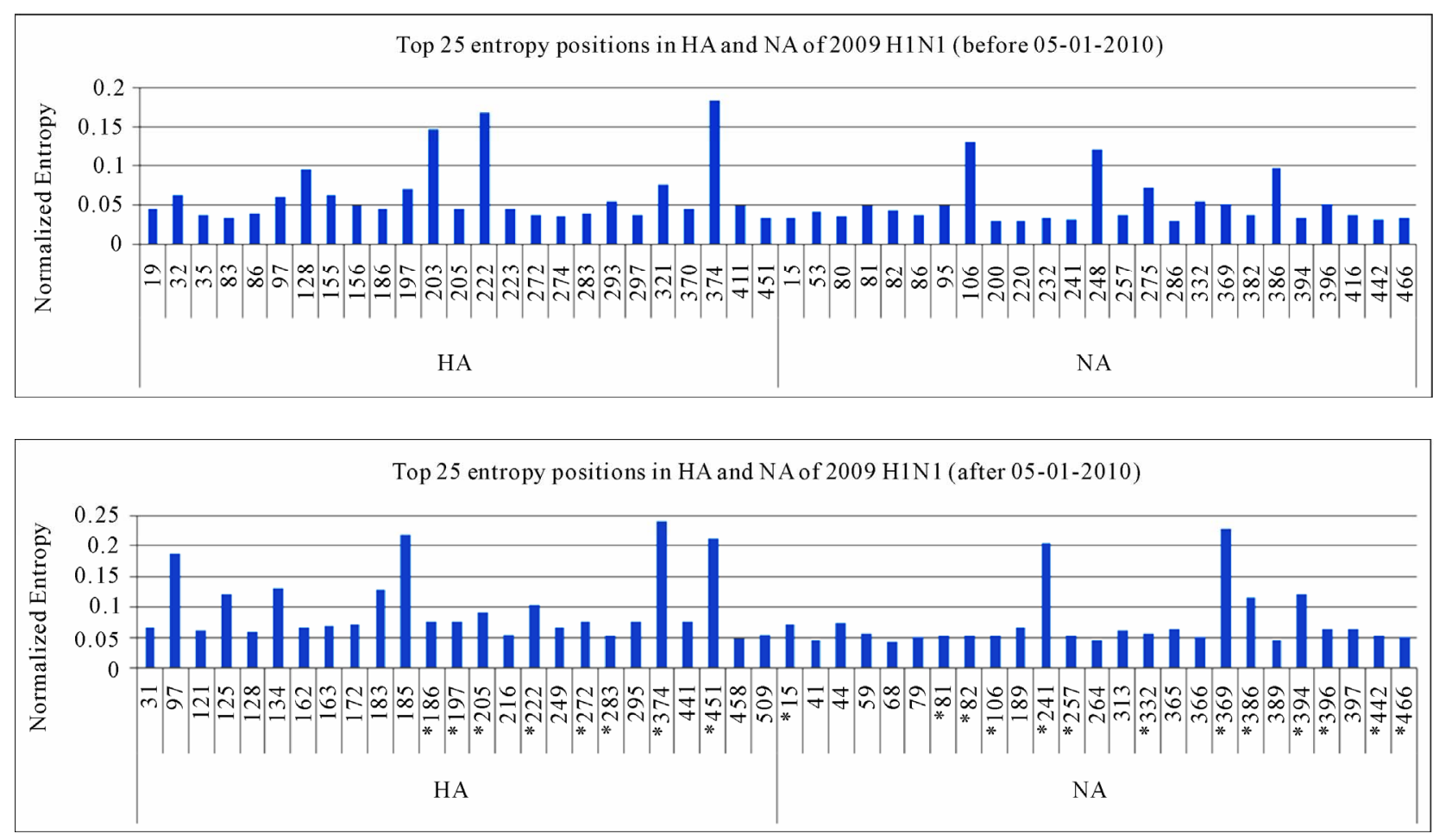

Figure 4. Top 25 entropy positions in the HA and NA of 2009 H1N1 before and after 05-01-2010. The positions marked with an asterisk in the bottom plot were selected both before and after 05-01-2010.

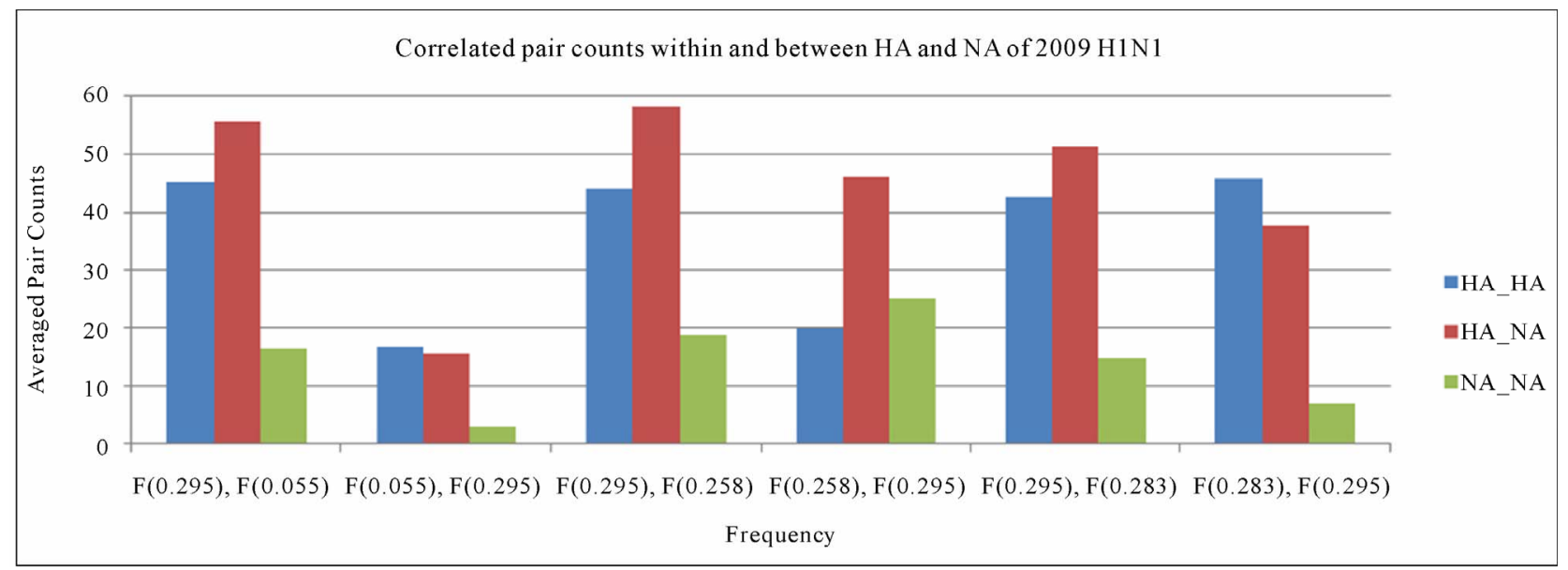

Figure 5. Correlation patterns of HA and NA of 2009 H1N1 before and after the HA receptor binding preference switch. In each group, the first frequency is primary and the second is secondary.

In the three cases of $\mathrm{F}(0.295)$ being the primary frequency, the correlation patterns were the same: the inter-protein correlation was higher than the intra-protein correlation and the correlation in HA was higher than in NA. However, after the primary frequency $F(0.295)$ being replaced by another, this pattern was altered. In the case of $F(0.055)$ and $F(0.283)$ being the primary frequency, the correlation was increased in HA and decreased in NA when compared to the interaction between $\mathrm{HA}$ and NA. In contrast, when $\mathrm{F}(0.258)$ was the primary frequency, the correlation was increased in NA and decreased in HA. These changes implied that the biological process of receptor selection shift could influence the collaboration between HA and NA.

\section{CONCLUSIONS}

This study focused on finding the mutations in the HA protein of $2009 \mathrm{H} 1 \mathrm{~N} 1$ collected from 05-01-2010 to 02-22-2011, and comparing them to those before 05-01- 
Table 4. Meta information for the HA and NA sequences in each group used to generate the graph in Figure 5.

\begin{tabular}{cccc}
\hline $\begin{array}{c}\text { Group } \\
\text { Number }\end{array}$ & $\begin{array}{c}\text { Primary } \\
\text { Frequency }\end{array}$ & $\begin{array}{c}\text { Secondary } \\
\text { Frequency }\end{array}$ & Size \\
\hline Group 1 & 0.295 & 0.055 & 333 \\
Group 2 & 0.055 & 0.295 & 24 \\
Group 3 & 0.295 & 0.258 & 655 \\
Group 4 & 0.258 & 0.295 & 28 \\
Group 5 & 0.295 & 0.282 & 143 \\
Group 6 & 0.282 & 0.295 & 13 \\
\hline
\end{tabular}

2010. The corresponding mutations in NA were also found. Our main strategy was to apply affinity propagation to discover six exemplars from the HA sequences of 2009 H1N1 after 05-01-2010. These six exemplars representing six clusters formed the basis for determining nine mutations in HA and four in NA. For each cluster, the unique combination of its primary HA binding IS frequency and secondary frequency were computed, reflecting the contribution of these nine mutations to receptor selection. Using a large number of 2009 H1N1 sequences available, mutual information analysis demonstrated the change of correlation patterns for the HA and NA of 2009 H1N1 in the process of HA receptor binding shift, thus highlighting the interdependence of these two surface proteins.

\section{ACKNOWLEDGEMENTS}

We thank Houghton College for its financial support and Naomi Komadina at WHO Research Centre in Australia for help with EpiFlu Database.

\section{REFERENCES}

[1] Mo, H. (2011) A/H1N1 now predominant flu strain in China. http://news.xinhuanet.com/english2010/china/ 2011-02/10/c 13726154.htm

[2] Barr, I.G., Cui, L., Komadina, N., Lee, R.T., Lin, R.T., Deng, Y., Caldwell, N., Shaw, R. and Maurer-Stroh, S. (2010) A new pandemic influenza A(H1N1) genetic variant predominated in the winter 2010 influenza season in Australia. New Zealand and Singapore. Europe Surveillance, 15, 19692.

[3] Hu, W. (2009) Analysis of correlated mutations, stalk motifs, and phylogenetic relationship of the 2009 influenza a virus neuraminidase sequences. Journal of Biomedical Science and Engineering, 2, 550-558. doi:10.4236/jbise. 2009.27080

[4] Hu, W. (2010) The interaction between the 2009 H1N1 Influenza a hemagglutinin and neuraminidase: mutations, comutations, and the na stalk motifs. Journal of Biomedical Science and Engineering, 3, 1-12.

[5] Hu, W. (2010) Novel host markers in the 2009 pandemic
H1N1 influenza a virus. Journal of Biomedical Science and Engineering, 3, 584-601.

doi: $10.4236 /$ jbise. 2010.36081

[6] Hu, W. (2010) Nucleotide host markers in the influenza a viruses. Journal of Biomedical Science and Engineering, 3, 684-699. doi:10.4236/ibise.2010.37093

[7] Hu, W. (2010) Identification of highly conserved domains in hemagglutinin associated with the receptor binding specificity of influenza viruses: 2009 H1N1, avian H5N1, and swine H1N2. Journal of Biomedical Science and Engineering, 3, 114-123. doi: $10.4236 /$ jbise. 2010.32017

[8] $\mathrm{Hu}, \mathrm{W}$. (2010) Quantifying the effects of mutations on receptor binding specificity of influenza viruses. Journal of Biomedical Science and Engineering, 3, 227-240. doi:10.4236/jbise.2010.33031

[9] Hu, W. (2010) Subtle differences in receptor binding specificity and gene sequences of the 2009 pandemic $\mathrm{H} 1 \mathrm{~N} 1$ influenza virus. Advances in Bioscience and Biotechnology, 1, 305-314. doi:10.4236/abb.2010.14040

[10] $\mathrm{Hu}, \mathrm{W}$. (2010) Correlated mutations in the four influenza proteins essential for viral RNA synthesis, host adaptation, and virulence: NP, PA, PB1, and PB2. Natural Science, 2, 1138-1147. doi:10.4236/ns.2010.210141

[11] $\mathrm{Hu}, \mathrm{W}$. (2010) Highly conserved domains in hemagglutinin of influenza viruses characterizing dual receptor binding. Natural Science, 2, 1005-1014. doi:10.4236/ns.2009.29123

[12] Hu, W. (2010) Host markers and correlated mutations in the overlapping genes of Influenza Viruses: M1, M2; NS1, NS2; and PB1, PB1-F2. Natural Science, 2, 1225-1246. doi: 10.4236/ns.2010.211150

[13] Hu, W. (2011) Receptor binding specificity and origin of 2009 H1N1 pandemic influenza virus. Natural Science, 3, 234-248. doi: 10.4236/ns.2011.33030

[14] Maurer-Stroh, S., Lee, R.T., Eisenhaber, F., Cui, L., Phuah, S.P. and Lin, R.T. (2010) A new common mutation in the hemagglutinin of the 2009 (H1N1) influenza a virus. PLoS Currents, 1, 1162.

[15] Brendan, J.F. and Delbert, D. (2007) Clustering by passing messages between data points. Science, 315, 972-976. doi:10.1126/science. 1136800

[16] Childs, R.A., Palma, A.S., Wharton, S., et al. (2009) Receptor-binding specificity of pandemic influenza A (H1N1) 2009 virus determined by carbohydrate microarray. Nature Biotechnology, 27, 797-799. doi:10.1038/nbt0909-797

[17] Katoh, K., Kuma, K., Toh, H. and Miyata, T. (2005) MAFFT version 5: improvement in accuracy of multiple sequence alignment. Nucleic Acids Reseach, 33, 511-518. doi:10.1093/nar/gki198

[18] Inmar, E.G. and Brendan J.F. (2009) A binary variable model for affinity propagation. Neural Computation, 21, 1589-1600. doi:10.1162/neco.2009.05-08-785

[19] Inmar, E.G. and Brendan, J.F. (2009) A binary variable model for affinity propagation. Neural Computation, 21, 1589-1600. doi:10.1162/neco.2009.05-08-785

[20] Delbert, D., Brendan, J.F., Nebojsa, J., Vladimir, J., et al. (2008) Constructing treatment portfolios using affinity propagation. International Conference on Research in Computational Molecular Biology, Singapore, 30 March - 
2 February 2008, 360-371.

[21] Delbert, D. and Brendan, J.F. (2007) Non-metric affinity propagation for unsupervised image categorization. International Conference on Computer Vision, Rio de Janeiro, 14-20 October 2007, 1-8.

[22] Nevena, L., Inmar, E.G., Parham, A. and Brendan, J.F. (2009) Facility location for subspace segmentation. 12th International Conference on Computer Vision, Kyoto, 29 September - 2 October, 2009, 825-832.

[23] Veljko, V., Henry, L.N., Sanja, G., Nevena, V., Vladimir, P. and Claude, P.M. (2009) Identification of hemagglutinin structural domain and polymorphisms which may modulate swine H1N1 interactions with human receptor. BMC Structural Biology, 9, 62. doi:10.1186/1472-6807-9-62

[24] Veljkovic, V., Veljkovic, N., Muller, C.P., Müller, S., SGlisic, S., Perovic, V. and Köhler, H. (2009) Characterization of conserved properties of hemagglutinin of $\mathrm{H} 5 \mathrm{~N} 1$ and human influenza viruses: Possible consequences for therapy and infection control. BMC Structural Biology, 7, 9-21.

[25] Veljkovic, N., Glisic, S., Prljic, J., Perovic, V., Botta, M. and Veljkovic, V. (2008) Discovery of new therapeutic targets by the informational spectrum method. Current Protein Peptide Science, 9, 493-506. doi: $10.2174 / 138920308785915245$

[26] Cosic, I. (1997) The resonant recognition model of macromolecular bioreactivity: Theory and application. Birkhauser Verlag, Berlin.

[27] Veljkovic, V., Veljkovic, N., Este, J.A., Huther, A. and Dietrich, U. (2007) Application of the EIIP/ISM bioinformatics concept in development of new drugs. Current Medicinal Chemistry, 14, 441-453. doi:10.2174/092986707779941014

[28] Cover, T.A. and Thomas, J.A. (1991) Elements of information theory. John Wiley and Sons, New York. doi:10.1002/0471200611

[29] David, M. (2003) Information theory, inference, and learning algorithms. Cambridge University Press, Cambridge.

[30] Andrea, K., Gabriel, R.N., Ivan, K.H. and Sccarontefan, J. (2002) Sequence similarities and evolutionary relationships of influenza virus A hemagglutinins. Virus Genes, 24, 57-63. doi:10.1023/A:1014038120979

[31] Le, L., Lee, E.H., Hardy, D.J., Truong, T.N. and Schulten, K. (2010) Molecular dynamics simulations suggest that electrostatic funnel directs binding of Tamiflu to influenza N1 neuraminidases. PLoS Computational Biology, 6, e1000939. doi:10.1371/journal.pcbi.1000939

[32] Sung, J.C., Van Wynsberghe, A.W., Amaro, R.E., Li, W.W. and McCammon, J.A. (2010) Role of secondary sialic acid binding sites in influenza N1 neuraminidase. Journal of American Chemical Society, 132, 2883-2885. doi:10.1021/ja9073672

[33] Zhou, H., Yu, Z., Hu, Y., Tu, J., Zou, W., et al. (2009) The special neuraminidase stalk-motif responsible for increased virulence and pathogenesis of H5N1 influenza A virus. PLoS One, 4, 6277. doi:10.1371/journal.pone.0006277

[34] Matsuoka, Y., Swayne, D.E., Thomas, C., Rameix-Welti, M.A., Naffakh, N., et al. (2009) Neuraminidase stalk length and additional glycosylation of the hemagglutinin influence the virulence of influenza H5N1 viruses for mice. Journal of Virology, 83, 4704-4708.

doi:10.1128/JVI.01987-08 